\title{
2 Research Square \\ Triplicate renal biopsies using core-cutting needles of different gauges in dogs
}

\section{Leandro Zuccolotto Crivellenti}

Universidade de Franca

\section{Sofia Borin-Crivellenti}

Universidade Federal de Uberlandia

\section{Larissa Ayane do Nascimento BRaz ( $\sim$ larissa-ayane@hotmail.com )}

Universidade Estadual Paulista Julio de Mesquita Filho Faculdade de Ciencias Agrarias e Veterinarias

Campus de Jaboticabal https://orcid.org/0000-0002-2522-8437

\section{Suellen Rodrigues Maia}

Universidade de Franca

Luciano Alves Farias

Universidade de Franca

Daniel Peixoto Pereira

Universidade de Franca

\section{Research article}

Keywords: complications, dogs, hematuria, glomeruli, renal disease

Posted Date: September 16th, 2019

DOl: https://doi.org/10.21203/rs.2.14512/v1

License: (c) (i) This work is licensed under a Creative Commons Attribution 4.0 International License.

Read Full License 


\section{Abstract}

Renal biopsy plays an important role in the diagnosis and management of renal disease, and core needle biopsy remains one of the most important investigation tools for this purpose in dogs. The size and quality of the biopsy material are likely to be important factors in achieving an accurate diagnosis. The aim of this study was to compare the success and complication rates of renal biopsy procedures performed in triplicates using needles of different gauges during open surgery. RESULTS: One hundred fifty-six biopsy samples were obtained from 52 dogs. Renal biopsies were performed using semiautomated biopsy needles of three different gauges, and subjected to histopathological examination and evaluation of complications. The $14 \mathrm{G}$ needle produced more severe hematomas than the $18 \mathrm{G}$ and $16 \mathrm{G}$ needles $(P=0.019)$. The longest post-biopsy microscopic hematuria observed ceased within five days after the procedure. Microscopic hematuria lasted longer in animals subjected to biopsy using $18 \mathrm{G}$ needles. There were no significant differences between needle gauges regarding macroscopic hematuria and number of erythrocytes in the urine immediately after biopsy. The numbers of glomeruli retrieved using 14G $(29.8 \pm 26.5)$ or $16 \mathrm{G}(27.1 \pm 14.6)$ needles differed from those retrieved using $18 \mathrm{G}$ needles (17.5 \pm 9.3$)$. CONCLUSION: The three needle gauges tested in this study are safe for use in renal biopsy using a semi-automated biopsy gun. The thicker needles provide more glomeruli, and, therefore, are more useful for diagnostic purposes. The use of $14 \mathrm{G}$ needles may be associated with more complications, and the $16 \mathrm{G}$ needle appears to offer the best compromise between diagnostic usefulness and biopsy-related complications.

\section{Background}

In patients with renal disease, renal biopsy provides a tool to determine the cause of the disease, predict the prognosis, and direct treatment. Histological examination of renal biopsy specimens remains the gold standard for diagnosis of kidney diseases $[3 ; 6 ; 8 ; 14]$. Although needle renal biopsy is considered a safe procedure [12;13;14], complications can occur and are mainly related to bleeding [3]. It is logical that using larger gauge needles would increase the number of glomeruli available for evaluation, but doing that would concurrently increase the risk of complications, especially hemorrhage. Similarly, experience of the individual obtaining the biopsy, severity or chronicity of disease, size of the patient, and a variety of other factors may affect not only the diagnostic yield of renal biopsy, but also complications associated with it [3,13]. As observed in humans [9; 10], canine renal biopsy samples show that an increase from 18 to 14 gauge $(G)$ is associated with significant increases in number of glomeruli per biopsy core [3; 11]. Bleeding, however, is more severe in human patients undergoing ultrasound-guided renal biopsies 
using $14 \mathrm{G}$ needles compared to those subjected to the same procedure using $18 \mathrm{G}$ needles [7]. These results have not yet been replicated in veterinary medicine, as most studies on this topic consist of retrospective analyses, or have not checked for complications in the same manner as described for humans [3].

In most cases, appropriate evaluation of a renal biopsy specimen taken from a dog with glomerular disease must include light, electron, and immunofluorescence microscopy [8;12] Light microscopy analysis requires that at least 2-3 samples are available to allow accurate histopathological interpretation $[1 ; 5]$ So far, no studies in veterinary medicine have evaluated the complications the patient may suffer as a consequence of renal biopsy, especially when this procedure is performed multiple times. Due to the lack of conclusive data on the effect of needle gauge on complications of renal biopsy in dogs, this prospective controlled study investigated the prevalence of hemorrhagic complication rates, ultrasound findings, and persistence of microscopic hematuria after biopsy was performed during open surgery with a semi-automated spring-loaded biopsy device. Additionally, biopsy sample quality and complication rates were compared between biopsy specimens obtained with 18G, 16G, or 14G needles.

\section{Results}

\section{Glomerular analysis}

Seven of 156 renal samples contained no glomeruli (4.49\%). Of these, 5 samples were obtained using $18 \mathrm{G}$ needles (71.4\%), one using a $14 \mathrm{G}$ needle $(14.3 \%)$, and one using a $16 \mathrm{G}$ needle (14.3\%). The total numbers of glomeruli harvested using 18G, 16G, and 14G needles were 17.5 $\pm 9.3,27.1 \pm 14.6$, and $29.8 \pm 26.5$, respectively ( $P=0.0457)$, as assessed by light microscopy. However, when the numbers of glomeruli per core were analyzed, there was no statistical difference among specimens harvested using 18G, 16G, and 14G needles $(11.9 \pm 7.0,14.1 \pm 8.8$, and $14.9 \pm 12.1$, respectively; $P=0.4051)$.

Weight was not a factor involved with bleeding $(P=0.439)$, hematuria $(P=0.279)$, hematoma ( $P=0.478)$, or number of glomeruli $(0.6322)$. Weight was not important for 
presence of medulla in the cores harvested $(P=0.1043)$ or number of glomeruli per core $(P$ $=0.8923)$.

Table 1. Mean number of glomeruli as assessed by light microscopy, and percentages of kidney samples with less than 10 glomeruli.

\begin{tabular}{lccccc}
\hline & $n$ & \multicolumn{2}{c}{ Light Microscopy } & $<10$ glomeruli & $<5$ glomeruli \\
\cline { 3 - 3 } & & $\begin{array}{c}\text { Mean number of } \\
\text { glomeruli }\end{array}$ & $\begin{array}{c}\text { Samples with } \\
\text { medullar tissue (\%) }\end{array}$ & LM (\%) & LM (\%) \\
\hline 18 & 21 & $17.5 \pm 9.3^{\mathrm{a}}$ & 33.3 & 9.5 & \\
Gauge & & & & 4.5 \\
16 & 25 & $27.1 \pm 14.6^{\mathrm{b}}$ & 28.0 & 12.0 & \\
Gauge & & & & 0 \\
14 & 6 & $29.8 \pm 26.5^{\mathrm{b}}$ & 16.0 & 16.7 & \\
Gauge & & & & \\
\hline
\end{tabular}

Mean values followed by different superscript letters in the same columns are statistically different $(p<0.05)$. LM, light microscopy.

\section{Medulla}

The percentage of renal biopsy samples that contained renal medulla fragments was similar between specimens harvested using 18G, 16G, and 14G needles ( $P=0.5862)$. 


\section{Crushes}

Kidney biopsies were considered adequate (good or excellent) by the histopathologist after light microscopy examination of the samples in $57.1 \%$ (18G), $72.0 \%$ (16G), and $66.7 \%$ (14G) of the specimens. Although higher amounts of fragmentation and crushing artifacts were observed in specimens harvested with an 18G needle, no statistical difference was observed between the three needle gauges in this regard $(P=0.8738)$.

Material for immunofluorescence was successfully obtained in 90.5\%, 96.0\%, and $83.3 \%$ of the cores harvested using 18G, 16G, and 14G needles, respectively. Material for electron microscopy was successfully obtained in $95.2 \%, 96.0 \%$, and $100 \%$ of the cores harvested using 18G, 16G, and 14G needles, respectively.

Table 2. Complications observed during execution of 156 renal biopsy procedures. Data on hematoma severity, microscopic hematuria, and persistence of microscopic hematuria are expressed as means \pm standard deviations. Data on macroscopic hematuria are expressed as percentages.

\begin{tabular}{lccc}
\hline & 18 Gauge & 16 Gauge & 14 Gauge \\
\hline Hematoma severity (0-3) & $0.82 \pm 0.81^{\mathrm{a}}$ & $0.71 \pm 0.78^{\mathrm{ab}}$ & $1.02 \pm 1.08^{\mathrm{b}}$ \\
Microscopic Hematuria (1-6) & $4.53 \pm 1.28$ & $4.02 \pm 1.45$ & $3.58 \pm 1.43$ \\
Macroscopic Hematuria (\%) & 21.7 & 20.0 & 16.7 \\
Persistence of microscopic hematuria (days) & $3.08 \pm 1.85^{\mathrm{a}}$ & $1.28 \pm 1.32^{\mathrm{b}}$ & $1.75 \pm 0.96^{\mathrm{ab}}$ \\
\hline
\end{tabular}

Mean values followed by different superscript letters in the same row are statistically different $(\mathrm{P}<0.05)$.

\section{Hematoma}

Hematoma was recognized immediately after harvesting the third core using 18G, $16 \mathrm{G}$, and $14 \mathrm{G}$ biopsy needles in $57.1 \%(\mathrm{n}=12), 48.0 \%(\mathrm{n}=12)$, and $83.3 \%(\mathrm{n}=5)$ of cases, respectively. Hematomas produced by $14 \mathrm{G}$ needles were more severe than those produced by $18 \mathrm{G}$ or $16 \mathrm{G}$ needles $(P=0.019)$. 


\section{Bleeding}

Macroscopic hematuria was observed in one dog in the $14 \mathrm{G}$ needle group (16.7\%), five dogs in the 16G needle group (20\%), and five dogs in the 18G needle group (21.7\%) $(P$ $=0.858)$. None of these episodes were associated with clot retention, and all cases subsided spontaneously after 24 hours. There was no requirement for blood transfusion or reoperation in any of the cases.

Microscopic hematuria was the most common consequence of the biopsy procedure (96.1\% of cases), and the patient could be followed at home using urinalysis dipsticks during 7 days in $71.2 \%(n=37)$ of the cases. The longest case of postbiopsy microscopic hematuria lasted for 5 days. There were no significant differences in number or erythrocytes in the urine immediately after biopsy using any of the needle gauges ( $P=$ 0.417), but persistence of postbiopsy microscopic hematuria was statically different after biopsy using the three biopsy needle gauges $(P=0.0264)$.

\section{Ultrasound}

An asymptomatic perirenal hematoma was detected by ultrasound 10 days after renal biopsy in one case (16G needle). Local tissue reaction was recognized nearby the biopsy site in 2 cases (18G and 16G needles), and presence of a small amount of liquid in the subcapsular area was detected in one case (14G needle). All the cases were managed conservatively with ultrasonographic reevaluation within 1 to 3 months.

Figure 1. Microscopic evaluation of renal biopsy using 18G, 16G, or 14G needles in 156 renal samples. Mean \pm standard deviation of the number of days of persistence of microscopic hematuria (A), hematuria severity score (B), total number of glomeruli obtained by biopsy (C), and glomerular crushing score (D). Asterisks indicate statistical differences between the groups evaluated.

\section{Discussion}

This study is, to our knowledge, the first trial to compare the safety and effectiveness of 14G, 16G, and 18G core-cutting needles in triplicate biopsies in dogs in a prospective 
fashion. The 14G needle group could be considered small. This fact likely reflects clinical practice and indications for renal biopsy as well as information from the latest studies that showed that $14 \mathrm{G}$ and $16 \mathrm{G}$ biopsy needles do not affect the number of glomeruli in biopsy specimens [3].

The three biopsy cores obtained from each animal were taken from the middle aspect of the kidney. This made the biopsy sample a good representative of the renal tissue, as the specimen would contain predominantly renal cortex tissue, and yield the maximum number of glomeruli [13].

The most important requirement for any biopsy procedure is that it should be safe and have a low incidence of clinically significant hemorrhage. The macroscopic hematuria frequency observed in this study (20.4\% of the cases) was greater than those described in other studies $(1-4 \%)[12 ; 13]$. It is possible that this difference is due to the fact that hematuria was assessed 24-48 hours after kidney biopsy in most other studies [14], while it was assessed right after the procedure in this study. This complication may upset the owner; however, maintaining the dog at the hospital for 24 hours after renal biopsy could minimize this problem, as macroscopic hematuria subsided spontaneously within 24 hours in the cases described in this study. In this trial, there were no significant differences between the three core-cutting needle gauges used regarding hematuria as a complication of the biopsy procedure, which suggests that even larger needles can be safe from this standpoint. Worse bleeding complications were not observed in this study, but were described elsewhere $[3 ; 14]$.

Veterinarians may be reluctant to use $14 \mathrm{G}$ or $16 \mathrm{G}$ needles for renal biopsy because they might be expected to cause more complications than thinner needles. Our findings partly support this assumption, as $14 \mathrm{G}$ needles were associated with greater hematoma severity than 18G and 16G needles. Importantly, all the other complication parameters were similar, independent of needle gauge. We suggest that biopsy route is more relevant for the occurrence of complications than the gauge of the needle used. The same has been shown in renal biopsy in humans [10]. 
A previous study found that renal biopsy performed during open surgery is surprisingly associated with greater amounts of medullar tissue in the specimens harvested when compared to ultrasound-guided biopsy [3]. In this study, we could not replicate those results, as needle biopsy performed during open surgery exhibited reduced penetration into the renal medulla. This difference could be explained by the fact that the needle is often inserted too deeply when biopsy is performed during open surgery, thus missing the cortex. Probably, that can happen owing to a tendency to reach the outer cortex and, then, insert the needle a little deeper "to make sure it is well inserted". Therefore, that last push makes the needle get past the cortex $[3 ; 8]$.

Dipsticks appear to be easy and cheap tools to monitor the patients for microscopic hematuria. Although the results obtained using dipsticks did not change the treatment in the cases described in this study, it could help to detect persistent bleeding complications. Future studies on the diagnostic value of this type of test are necessary.

A persistent silent perirenal hematoma was found in only one case $(1.8 \%)$ in this study. This frequency is lower than that reported in humans $(65 \%)[15 ; 16]$. Possible reasons for hematoma variability include timing of ultrasonographic examination [15], and diligence of the search [2] and imaging methods [16]. It is likely that the low number of hematomas found in this study is due to the time at which the ultrasound examination was conducted (only 10 days after the procedure). The optimal timing of scanning to detect hematomas and predict complications is not known, but ultrasonographic examination at 10 days after the procedure appears to be appropriate for biopsies performed during open surgery because, under these conditions, bleeding complications can be easily visualized right after biopsy. Perhaps ultrasound imaging immediately to 48 hours could be more important after percutaneous kidney biopsy to find complications, as described [14]. In this study, other types of complication like subcapsular or perirenal hematomas, pseudoaneurysm, and arteriovenous fistula could not be found.

Samples from small animals have been shown to be affected by needle gauge [14]; specially, in small breeds. However, in this work, neither number of glomeruli nor the 
frequency of samples with presence of medullar tissue in the cores was verified. Probably the results difference is due to the fact of small breeds have renal thin cortex. We suggest that short-throw (first-stage) biopsy needles could be more appropriate in those cases.

The thicker 14G and 16G biopsy needles provide greater tissue yield and number of glomeruli than the thinner 18G needles. The 18G biopsy needles, albeit thought to be less invasive, provide insufficient tissue, relatively poor sample quality, and, apparently, are not safer than thicker needles, especially 16G needles. The use of 16G needles provides the best compromise between diagnostic usefulness and patient safely.

\section{Conclusion}

Our analysis finds that the risk of hemorrhagic complications is low if the appropriate technique is applied. Urine evaluation by the patient's owner during the first days after renal biopsy can be a cheap and easy method, and postbiopsy ultrasound scanning can be useful. The three needle gauges tested in this study are safe for use in renal biopsy using a semi-automated biopsy gun. The thicker needles (14G and 16G) provide more glomeruli, and, therefore, are more useful for diagnostic purposes. The use of $14 \mathrm{G}$ needles may be associated with more complications, and the 16G needle appears to offer the best compromise between diagnostic usefulness and biopsy-related complications.

\section{Methods}

One hundred fifty-six biopsy samples were obtained from 52 dogs. Biopsies were performed using semi-automated biopsy needles (M.D.L aghi specialli e componenti medicali - Italy) of three different gauges. A single operator performed the biopsy procedure. The operator chose between the 16G and 18G randomly for breeds of all sizes, and used 14G needles for medium and large breeds. A completely randomized approach was not performed because some veterinary studies recommend not to use large needles in small dogs (< $10 \mathrm{~kg}$ ) [8;14]; additionally, there is no difference in number of glomeruli between samples obtained using $16 \mathrm{G}$ or $14 \mathrm{G}$ needles [3]. 
All the biopsies were carried out during open surgery for better evaluation of complications. Light, immunofluorescence, and electron microscopy were performed as part of another study to investigate subclinical proteinuria in different glomerular diseases in routine patients. The following inclusion criteria were used, to avoid factors that could affect in sample quality and complications rates: creatinine within the normal range $(<1.4$ $\mathrm{mg} / \mathrm{dL}$ ), normoalbuminuria, proteinuria within non-nephrotic range, and absence of important laboratorial alterations.

Renal biopsies were performed with patients in dorsal recumbency during spaying surgery. No imaging guidance was needed or used for this experimental study; the needle was advanced horizontally for a few millimeters into the renal cortex under direct visualization, and then biopsy samples were obtained from an intact cortical surface. The two-stage tissue cutting action of the biopsy needle was activated.

Samples were obtained from the middle aspect of the left kidney. All the cores obtained were collected regardless of the gross quality of the sample; triplicate biopsies were performed on all animals, as this is standard practice in various institutions $[3,4,5]$. Cores were removed from the shaft of the needle by gentle tapping of the needle tip immersed within saline solution for subsequent sectioning. For histopathological examination, the biopsy specimens were divided into samples for light, immunofluorescence, and electronic microscopy.

Samples were preferentially reserved for light microscopy and immunofluorescence. Two small fragments of the third biopsy specimen were reserved for electron microscopy, while the remaining tissue fragment was reserved for light microscopy.

Following the biopsy, firm pressure was applied to the needle entry site during 3-5 minutes. An auxiliary surgeon blinded to the needle gauge used provided an assessment of bleeding severity (mild, moderate, or severe) and size of hematomas (none, small, medium, or large) after the procedure was completed. 
After the biopsy procedure, the patients were monitored closely; a postbiopsy urine sample was collected, and presence or absence of macroscopic hematuria was recorded. If no macroscopic hematuria was found, presence of erythrocytes was assessed by microscopy and categorized in a scale from 1 to 6 , in which 1 represented less than 5 erythrocytes per high power field, and 6 denoted TNTC (too numerous to count). Presence of microscopic hematuria was evaluated using dipsticks every 24 hours during 7 days by the patients' owners at home. Only presence or absence of occult blood was analyzed statically because the intensity of color in dipsticks could be perceived - and reported differently by the owners and, therefore, skew the results.

All paraffin sections were examined by two researchers who were blinded to the type of the needle used. The quality of the material was categorized according to a score system (poor, good, or excellent).

Doppler ultrasound examination was performed a few days before the renal biopsy, and was repeated 10 days after the procedure. If necessary, an additional Doppler ultrasound exam was performed 1-3 months later to search for evidence of hydronephrosis, hematoma, or infection of the biopsy site that might have developed after the biopsy procedure.

Results were subjected to statistical analysis to compare mean values; the multiple comparison Tukey-Kramer test was used. Differences were considered significant at P < 0.05. Calculations were performed using JMP ${ }^{\circledR}$ software.

\section{Declarations}

\section{Acknowledgments}

Not applicable. 
LZC and SBC designed the experiments, analyzed the data and drafted the manuscript together. PW, YL and HT performed the experiments. LANB, SRM, LAF and DPP helped to draft the manuscript. All authors read and approved the final manuscript.

\section{Availability of data and materials}

The datasets used and/or analysed during the current study are available from the corresponding author on reasonable request.

\section{Funding}

Dr. Crivellenti was supported by a scholarship from the Fundação de Amparo à Pesquisa do Estado de São Paulo (grant no. 2014/21506-2).

\section{Ethics approval and consent to participate}

All the procedures were approved by the Veterinary Ethics Committee of UNESP Universidade Estadual Paulista, Jaboticabal, Brazil (011862-11). The animals were included after informed client consent.

\section{Consent for publication}

Not applicable.

Competing interests

The authors declare that they have no competing interests.

\section{References}

1. Cianciolo RE, Brown CA, Mohr FC, et al. Pathologic evaluation of renal biopsies: Methods for identifying features that differentiate immune-mediated 
glomerulonephritides from other categories of glomerular disease. J Vet Intern Med 2013;27: S10-S18.

2. Corapi KM, Chen JL, Balk EM, Gordon CE. Bleeding complications of native kidney biopsy: a systematic review and meta-analysis. Am J Kidney Dis 2012; 60: 62-73.

3. Crivellenti LZ, Cianciolo R., Wittum T, Lees GE, Adin CA. Associations of patient characteristics, disease stage, and biopsy technique with the diagnostic quality of core needle renal biopsy specimens from dogs with suspected kidney disease. Am Vet Med Assoc2018; 252(1), 67-74.

4. Donnelly S, Goodyer P, Mauer M; RASS Investigators. Comparing the automated versus manual method of needle biopsy for renal histology Artefacts. Nephrol Dial Transplant 2008; 23: 2098-100.

5. Hussain F, Mallik M, Marks SD, Watson AR. British Association of Paediatric Nephrology: Renal biopsies in children: Current practice and audit of outcomes. Nephrol Dial Transplant 2010; 25:485-489.

6. Karam AR, Nugent W, Khan A, Desai D, Shankar S. Curved stylet core biopsy results in larger cores. AJR Am J Roentgenol. 2010;195:242-4

7. Kim D, Kim H, Shin G, Ku S, Ma K, Shin S, Gi H, Lee E, Yim H. A randomized, prospective, comparative study of manual and automated renal biopsies. Am J Kidney Dis 1998;32:426-431.

8. Lees GE, Cianciolo RE, Clubb FJ. Renal biopsy and pathologic evaluation of glomerular disease. Top Companion Anim Med 2011;26:143-153.

9. Mai J, Yong J, Dixson H, Makris A, Aravindan A, Suranyi MG, Wong J. Is bigger better? A retrospective analysis of native renal biopsies with 16 Gauge versus 18 Gauge automatic needles. Nephrology (Carlton) 2013;18:525-530.

10. Nicholson ML, Wheatley TJ, Doughman TM, White SA, Morgan JDT, Veitch PS, Furness PN. A prospective randomized trial of three different sizes of core-cutting needle for transplant biopsy. Kidney Int 2000;58:390-395.

11. Rawlings CA, Diamond H, Howerth EW, Neuwirth L, Canalis C. Diagnostic quality of percutaneous kidney biopsy specimens obtained with laparoscopy versus ultrasound 
guidance in dogs. J Am Vet Med Assoc 2003;223:317-321.

12. Vaden SL. Renal biopsy: methods and interpretation. Vet Clin North Am Small Anim Pract 2004;34:887-908.

13. Vaden, S.L. Renal biopsy of dogs and cats. Clin Tech Small Anim Pract. 2005;20:11-22

14. Vaden SL, Levine JF, Lees GE, et al. Renal biopsy: a retrospective study of methods and complications in 283 dogs and 65 cats. J Vet Intern Med 2005; 19(6):794-801.

15. Walker PD. The renal biopsy. Arch Pathol Lab Med 2009;133:181-188.

16. Whittier WL. Complications of the percutaneous kidney biopsy. Adv Chronic Kidney Dis 2012; 19: 179-187

\section{Figures}




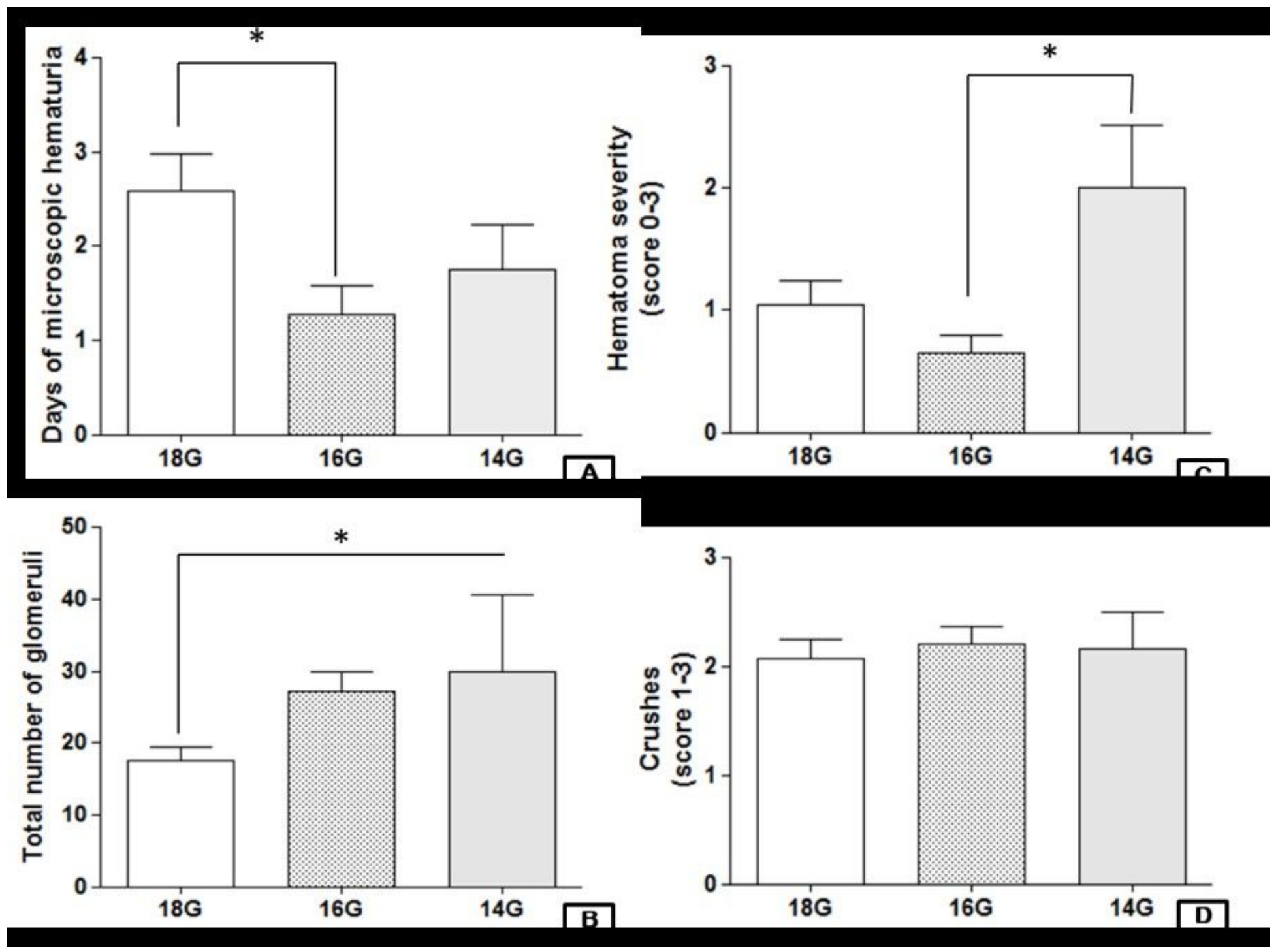

Figure 1

Microscopic evaluation of renal biopsy using 18G, 16G, or 14G needles in 156 renal samples. Mean \pm standard deviation of the number of days of persistence of microscopic hematuria (A), hematuria severity score (B), total number of glomeruli obtained by biopsy (C), and glomerular crushing score (D). Asterisks indicate statistical differences between the groups evaluated. 